\title{
Using Interactive Exercise in Mobile Devices to Support Evidence-based Teaching and Learning
}

\author{
M. Muztaba Fuad \\ Dept. of Computer Science \\ Winston-Salem State Uni. \\ Winston-Salem, NC, USA \\ +1-336-7503325 \\ fuadmo@wssu.edu
}

\author{
Debzani Deb \\ Dept. of Computer Science \\ Winston-Salem State Uni. \\ Winston-Salem, NC, USA \\ +1-336-7502496 \\ debd@wssu.edu
}

\author{
James Etim \\ Dept. of Education \\ Winston-Salem State Uni. \\ Winston-Salem, NC, USA \\ +1-336-7502382 \\ etimj@wssu.edu
}

\author{
Clay Gloster \\ Dept. of CST \\ NC A\&T State Uni. \\ Greensboro, NC, USA \\ $+1-336-3347717$ \\ cgloster@ncat.edu
}

\begin{abstract}
To improve student's class experience, the use of mobile devices has been steadily increasing. However, such use of mobile learning environments in the class is mostly static in nature through content delivery or multiple choice and true/false quiz taking. In CS courses, we need learning environments where students can interact with the problem in a hands-on-approach and instructor can assess their learning skills in real-time with problems having different degree of difficulty. To facilitate such interactive problem solving and real-time assessment using mobile devices, a comprehensive backend system is necessary. This paper presents one such system, named Mobile Response System (MRS) software, associated interactive problem-solving activities, and lessons learned by using it in the CS classrooms. MRS provides instructor with the opportunity of evidence-based teaching by allowing students to perform interactive exercises in their mobile devices with different learning outcomes and by getting an instant feedback on their performance and mental models. MRS is easy-to-use, extensible and can render interactive exercises developed by third-party developers. The student performance data shows its effectiveness in increasing student understanding of difficult concepts and the overall perception of using the software was very positive.
\end{abstract}

\section{Keywords}

Interactive learning environments; educational technology system; improving classroom teaching; STEM learning; mobile technology.

\section{INTRODUCTION}

Studies [1]-[2] have shown that in order to improve student learning in CS, traditional pedagogical approaches are not enough to transfer critical knowledge to students. More interactive teaching and learning strategies are necessary to make learning more productive. In CS courses, students need to actively solve problems by interacting with the problem in a hands-on approach.

Permission to make digital or hard copies of all or part of this work for personal or classroom use is granted without fee provided that copies are not made or distributed for profit or commercial advantage and that copies bear this notice and the full citation on the first page. Copyrights for components of this work owned by others than ACM must be honored. Abstracting with credit is permitted. To copy otherwise, or republish, to post on servers or to redistribute to lists, requires prior specific permission and/or a fee. Request permissions from permissions@acm.org.

ITiCSE '16, July 09-13, 2016, Arequipa, Peru

(C) 2016 ACM. ISBN 978-1-4503-4231-5/16/07 ..\$15.00

DOI: http://dx.doi.org/10.1145/2899415.2899467
Students cannot develop skills such as problem solving and critical thinking only by using traditional question types, such as multiple-choice or true-false questions. Since exams and quizzes are the dominant form of assessing student learning, we need to make such assessment tools more interactive and involving to gaze student learning, mental models and engagement better. We argue that by presenting the problems as interactive entities, where students can actively participate in different steps of the problem; student's critical thinking and problem solving skills can be improved. Mobile technology has brought incredible opportunities for educators to enable and deliver learning in ways that could not have been accomplished before. There have been an increasing number of studies [3]-[7] related to the research and development of learning environments intended for mobile computing devices. This study proposes having interactive exercises (IE) in the form of mobile App quizzes rather than traditional paper-based quizzes where the goal is to make the problem-solving exercises more visual and appealing to the students and allow them to realize the effect of their interactions at the different stages of the exercise. We expect the students to fully comprehend a concept and clarify any confusion through visual presentation, active interaction and hands-on nature of the mobile-based approach. As it is possible to automate the question generation, delivery, time keeping and grading in the proposed approach, we anticipate that the instructors would be able to offer more quizzes and students could have more opportunity to practice concepts where they receive immediate feedback.

Having IE activities as mobile Apps encourage evidence-based teaching practices as well. Research has shown that immediate feedback has positive effect on students' success [8]-[9] and by utilizing the proposed mobile-based approach, an instructor could provide immediate and context sensitive feedback to the students. Additionally, instructor would have the ability to identify and correct common misconceptions and reinforce specific topics immediately which students have not shown mastery of. By monitoring and analyzing student device usage data (button clicks, time spent, navigation behavior etc.) through these Apps, instructors have better understanding of their attitude, mental model, and barriers that they faced during problem solving.

To facilitate such interactive problem solving and real-time assessment and tracking using mobile devices, a comprehensive backend system is necessary. This paper presents details of Mobile Response System (MRS) software [10]-[12] and its usage and evaluation in the classroom. MRS facilitates anonymous communication, interaction and evaluation of in-class interactive problem solving activities using mobile devices. MRS enables a feedback-driven and evidence-based teaching methodology, 
which is important to enhance student learning. MRS is a clientserver software that allows the instructor to dynamically prompt the students with interactive exercises, synchronized with the lecture material in their mobile devices. Students are able to actively interact with the problem and send their answers back to the server computer. MRS then facilitates grading of the exercises automatically, by comparing the student made sequence of steps with the correct sequence of steps. After grading, MRS also makes the grading statistics and student submissions available for the instructor to view and share with the students.

This formative assessment allows the instructor to have real-time evidence of students' comprehension of covered lecture materials on a particular class and also helps instructor to identify the concepts that need to be repeated or reinforced. With MRS, instructor can also capture screens from student submissions and generate immediate discussion on those screens if contextsensitive feedback is needed. This approach allows the students to obtain faster and frequent feedbacks that reinforce their learning and help them to identify misconceptions and problem areas. The visual and active interaction with a problem via multiple steps while going back and forth and seeing the consequence of their choices at each step is expected to enhance students' analytical and problem solving capabilities. The other important feature supported by MRS is the ability to submit feedback/question anonymously during the class. Several studies [13]-[14] identifies the benefits of remaining anonymous during social interaction and the proposed approach leverages that in the mobile learning environment. The software allows students to send anonymous feedback/questions to the instructor and to vote on existing pool of questions that instructor may choose to review and answer at the end of the class. This encourages more class interaction and in-depth discussion between students and instructor. Finally, MRS is extensible to other disciplines than $\mathrm{CS}$ and can render interactive exercise Apps developed by third-party developers.

The rest of the paper is organized as follows: section two details the MRS system and interactive exercises, section three presents the evaluation data that is gathered when MRS is deployed in CS classrooms, section four discusses the related researches and section five concludes the paper.

\section{MRS SYSTEM}

The MRS software is designed as a client-server application. The instructor computer runs the server component of the software, which hosts questions, manages users, and maintain communication and synchronization. The client component executes in student's mobile devices and allows students to login to the system, to submit anonymous feedbacks/questions, and facilitates interactive exercise solving. The server has been developed in Java and the client has been developed in Android.

\subsection{MRS Client}

The client is a light-weight mobile application that provides essential functionality to deploy IE apps. It allows verification of student credentials and once logged in, the client shows a home screen where student can either submit anonymous feedback/question or exit from the system. When the client receives a new IE problem from the server, the corresponding App that renders the given problem into IE is located by the client and then executed. The MRS client and an IE activity App communicates using standard API calls and therefore is completely separated in application logic. This separation allows MRS's features to be extended to any domains with different IE Apps. More information about extensibility is provided in Section
2.4. Every IE activity has a set amount of time to answer (assigned by the instructor) and a visible timer starts counting for students to see how much time they have left to answer the question. Once the student answer is received, the client will capture that and will send it to the server for grading. At any time during the class, students can initiate a session from the home screen to post a feedback/question or vote on an existing one anonymously.

\subsection{MRS Server}

The MRS server is designed as a multi-process, multi-threaded entity to satisfy simultaneous invocation from users and to provide real time responses to in-class activities. Instructor running the MRS server can import student credentials, which will allow students to login to the sever. When a student logs in to the system, the server validates the identity and sets privileges accordingly. Once all students are logged in, instructor can import the IE question and broadcast them to the client devices. When the server receives answers back from all the clients, it uses runtime reflection to find the corresponding server-side grading component of the IE and loads and executes the corresponding method in that component dynamically in order to grade student submissions. As soon as grading is completed, MRS sends each student an email with the correct answer, their answer and their score in that exercise. This happens instantaneously and students receive the email with the feedback and their score in real time. Separate thread of the server constantly monitors whether a student using the client App wants to initiate a feedback/question session. In that case, it sends the current pool of feedback/questions to the corresponding client. Once a response from the client is received, if it is a new entry, then it is added to the existing list. Otherwise a vote on an existing entry is increased by matching it with the list of entries.

\subsection{Interactive Exercises (IE)}

The most important aspect of the MRS software is the facilitation of interactive problem solving. An interactive problem solving activity is defined as an Interactive Exercise (IE) with corresponding grading components and associated rubrics. In an IE, students require to directly work on a visual representation of a problem and develop the answer following a set of steps guided by a particular algorithm or process. In each step, students make key choices (for example clicking the table or array indices for selection or swap, selecting from a drop-down menu, selecting a tree node or an edge of a graph etc.) that will impact their next step of interaction. During these interaction steps, students can go back and forth (by utilizing "Back" and "Next" button) and see the impact of their different choices. Interactive exercises can be offered as solving a whole problem from bottom-up or top-down fashion or solving certain steps of a particular problem in order to give students different perspective on the problem and to assess their problem solving skills. Only after the student traverse each of the steps or the allotted time to answer a problem runs out, the results of their interactions performed at each step are then sent back to the server. The MRS software automatically handles the grading of the exercises, by comparing the student made sequence of interactions with the model sequence of interactions for a particular problem. Each problem has a rubric that grade partially correct answers to gauge student's problem solving skills and cognitive models.

In this research, the interactive exercises are designed as dynamic entities rather than static entities to support greater diversity and to allow students to practice the same problem solving activity with different question parameters. The exercises are therefore 
parameterized, where parameters can be populated with either randomly generated values or instructor generated values to create different instances of a problem. An IE definition is stored as an XML file and contains exercise parameters such as problem components, time to answer, special instructions etc., which are used to render the corresponding IE App in the client device. The file additionally contains different rubric and grading parameters such as correct answers, step-wise grading weights, etc. to support server-side automated grading. Student device usage during interactive problem solving can also be tracked by setting the correct parameters in the IE definition file such as how often they utilize the "Back" button, how much time they spend in individual stages of a problem, how often they navigate to other Apps (such as browsers, Tweeter, Facebook etc.). The data gathered on these aspects of the system shows some interesting insight into student learning and engagements. For instance, students barely switch to other Apps during problem solving sessions, which shows that they are completely engaged into problem solving and that they are not looking for answers in the internet. Also the more time a student presses back button, her grade is better on that problem compared to a student who did not utilizes back button frequently. This validates that the ability to go back-and-forth during problem solving enable students to check their actions better. To date, seven interactive exercise Apps have been developed as listed in Table 1.

Table 1. Developed Interactive Exercise (IE) Apps

\begin{tabular}{|c|c|}
\hline App & Where can be deployed? \\
\hline Interactive matching & Any course, Any discipline \\
\hline Analytical answering & Any course, Any discipline \\
\hline Truth Table formation & Freshman/sophomore CS/EE course \\
\hline K-map simplification & Freshman/sophomore CS/EE course \\
\hline Bubble Sorting & Sophomore/Junior CS course \\
\hline Selection Sorting & Sophomore/Junior CS course \\
\hline $\begin{array}{c}\text { Prim's Minimum } \\
\text { Spanning Tree }\end{array}$ & Sophomore/Junior CS course \\
\hline
\end{tabular}

Figure 1(a), (b) and (c) shows intermediate screens of an IE App that was developed for assessing the student's understanding of K-map simplification algorithm. At first, students can select cells corresponding to the given Sum of Minterm expression (Figure 1(a)). Correct execution of this step verifies whether students learned how the map is laid out and what every cell points to. Students then make groups with the cells following the minimization algorithm (Figure 1(b)) in the next screen. Students can select any cell and each group made will take a different color (with the group number as superscript) to distinguish it from other groups. This step validates whether students comprehend different attributes of making groups. Next, students interact with each group and select common literal from each group (Figure 1(c)) following the minimization algorithm. During these steps, student can traverse back and forth and change their answer. However, since the IE App cannot verify whether the student devised answer is correct or not; students can not heuristically figure out the answer of a question. Therefore, what the student come up as the answer of a problem, represents student's own understanding and learning levels.

The MRS server automatically grades student submitted exercises, summarizes them, and displays grading and tracking statistics for instructor to gain further insight and to share them with the class. Figure 2(a) captures the "Grade Summary" screen that instructor can immediately share with the class. The "Grade Details" tab presents student performance data for individual problem steps, so that instructor can reinforce some particular step of the problem immediately if majority students struggle on that step instead of repeating the whole process. The "Time Taken" tab displays step-

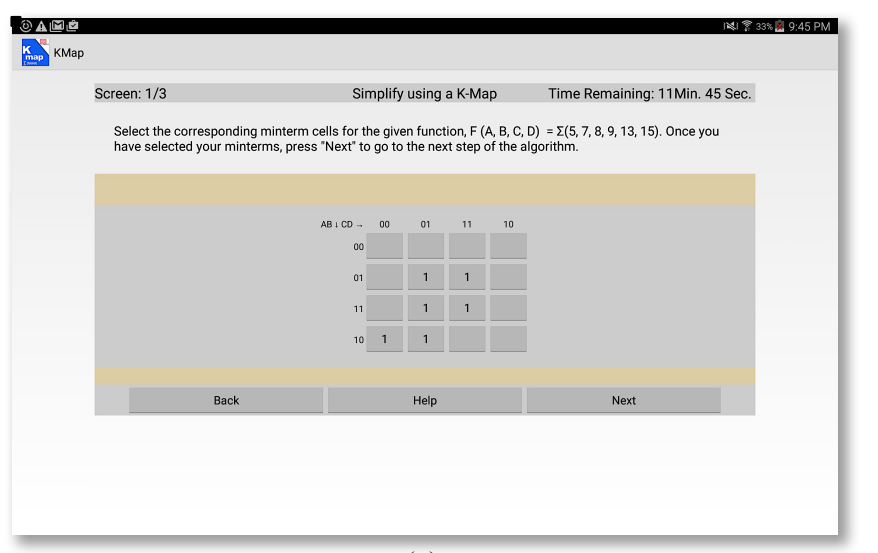

(a)

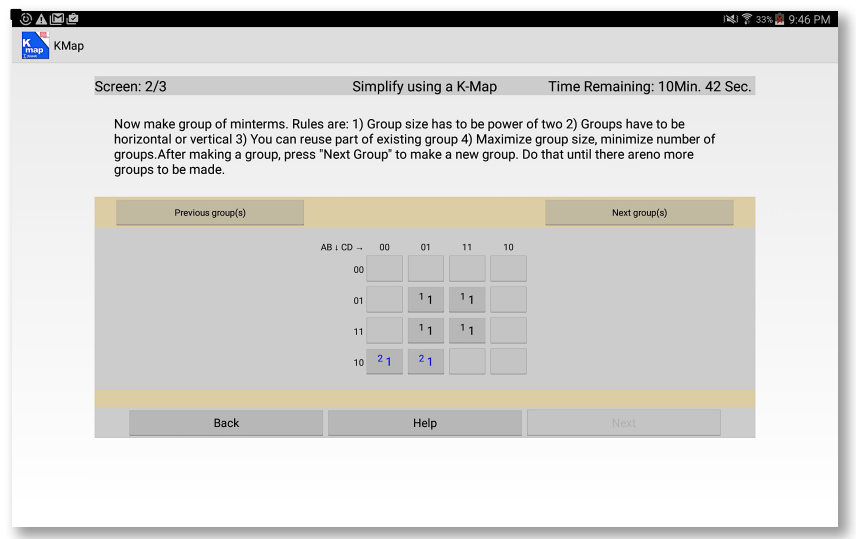

(b)

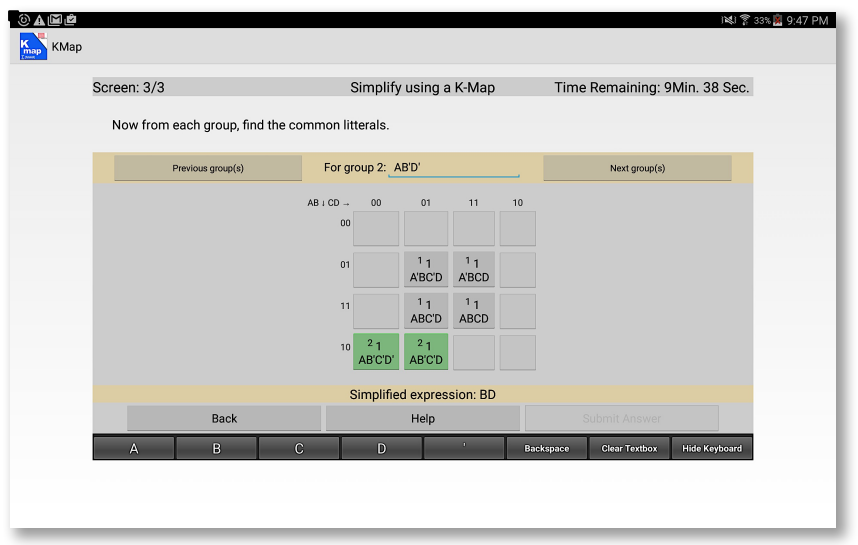

(c)

Figure 1. Intermediate screens of k-map App.

wise student timing data to understand the steps that students spend most time on. The "App Swap" tab tracks "Back" button usage and "Navigation" tab tracks how often student navigates away to other Apps during problem solving. Instructor can share the correct answer with the class by utilizing the "Correct Answer" tab. Moreover, each student receives an email instantly with an attachment (Figure 2(b)) that notifies them of their score with the correct answer and their answer of the exercise for future review. Immediate availability of this step-wise assessment and tracking information allows instructor to gain better understanding of student learning of a concept and allows students to assess their 
own progress toward the concept and to compare it with the rest of the class.

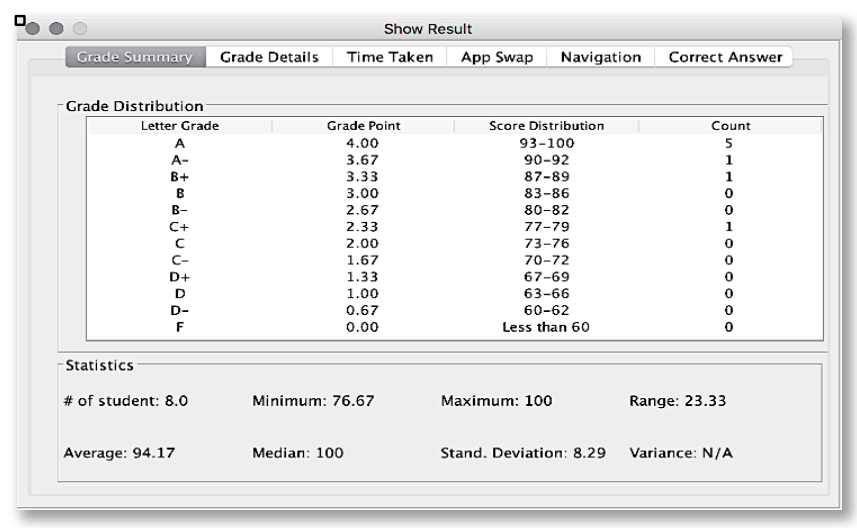

(a)
Correct Answer

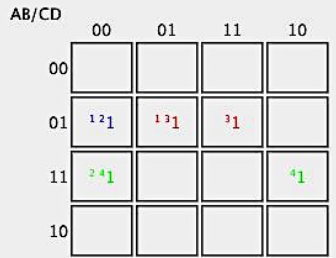

Simplified Expression: $B C^{\prime} D^{\prime}+A^{\prime} B D+A B D '$ or $A^{\prime} B C^{\prime}+A^{\prime} B D+A B D^{\prime}$

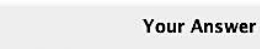

$\mathrm{AB} / \mathrm{CD}$

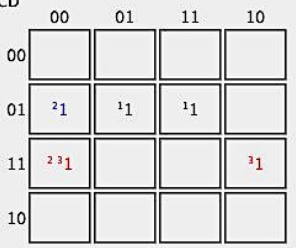

Simplified Expression: $\mathrm{A}^{\prime} \mathrm{BD}+\mathrm{BC}^{\prime} \mathrm{D}^{\prime}+\mathrm{ABD}$

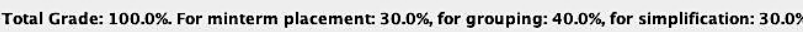
(b)

Figure 2. Grading statistics and grade notification email.

\subsection{MRS Extensibility}

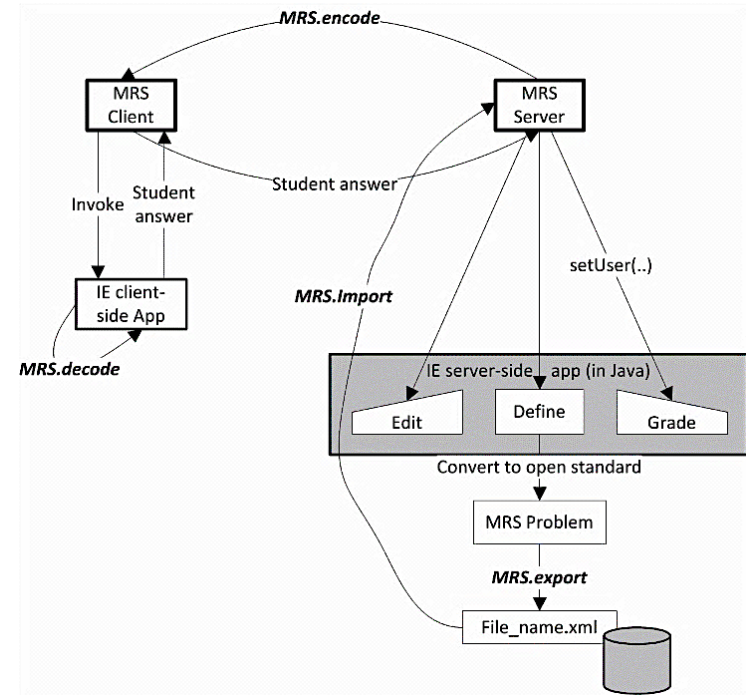

Figure 3. MRS components, interfaces, and interactions.

In order to encourage broader adoption and dissemination, MRS is designed to be extensible and therefore has the ability to render IE Apps created by third-party developers. To build a problemsolving interactive exercise App, the third-party developer needs to design the nature of interactions and implement Android's activity life cycle accordingly for their App. In order to incorporate that App to MRS environment, additionally they need to create the corresponding IE definition file and server-side grading component. Then by following a set of well-specified requirement and APIs (Figure 3), one can integrate and execute any IE App into MRS learning environment with minimal effort.

\section{EVALUATION}

The MRS software and associated interactive exercises were deployed in a sophomore course (traditional face-to-face classroom setting) titled as "Introduction to Computer Hardware Organization" during the period of Fall 2014, Spring 2015, and Fall 2015. In each class, students were given Android tablets to use during the class for interactive exercise solving and anonymous questions/feedback submission or for performing other class related works. Students are allowed to utilize their own tablet or mobile device as long as they have installed the MRS client. The current version of MRS only support Android clients, however the system is built in a way that accommodates client from any platforms. The evaluation of the impact of the MRS learning environment is determined using the data collected from an experience survey, from a focus group, and from a comparison of the grades received by students when completing targeted exercises with or without using the software. During Fall 2014, the deployment was carried out as a Pilot study for recognizing and correcting errors and to fine-tune the implementations. Therefore, the data gathered during this term is excluded from the evaluation. Table 2 shows some important class related attributes. In both intervened semesters, instructors were able to administer similar or more graded exercises compared to the non-intervened semesters. However, since the process of grading and management was automated, instructor could spend more time on answering student questions and encourage class discussions. The same exercises were offered in the class in a pen- and-paper based setting without MRS and in the mobile IE App setting with MRS. IEs related to two separate concepts, truth-table and k-Maps were used before and after MRS. For the sake of comparison, same weighted grade calculation was utilized in all semesters.

Table 2. Class related Information

\begin{tabular}{|l|c|c|c|c|}
\hline \multirow{2}{*}{} & \multicolumn{2}{|c|}{ Without MRS } & \multicolumn{2}{c|}{ With MRS } \\
\cline { 2 - 5 } & $\begin{array}{c}\text { Fall } \\
2013\end{array}$ & $\begin{array}{c}\text { Spring } \\
2014\end{array}$ & $\begin{array}{c}\text { Spring } \\
2015\end{array}$ & $\begin{array}{c}\text { Fall } \\
2015\end{array}$ \\
\hline \# of students & 11 & 17 & 14 & 8 \\
\hline \# of graded exercise & 11 & 10 & 11 & 13 \\
\hline $\begin{array}{l}\text { \# of anonymous } \\
\text { questions/feedbacks }\end{array}$ & N/A & N/A & 3 & 34 \\
\hline $\begin{array}{l}\text { \# of votes on } \\
\text { questions/feedbacks }\end{array}$ & N/A & N/A & 4 & 53 \\
\hline
\end{tabular}

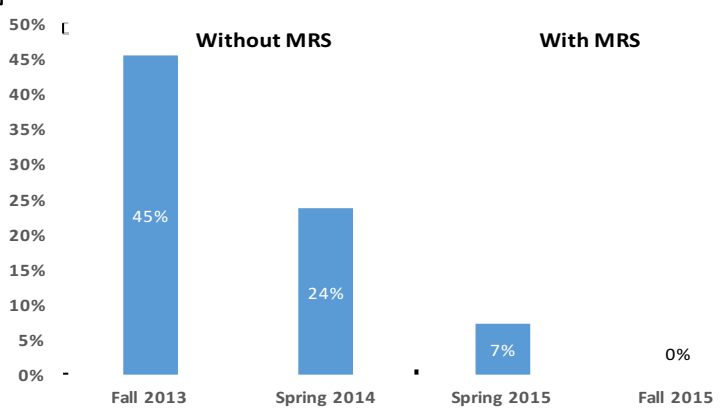

Figure 4. \% of D's and F's with and without MRS.

Figure 4 presents the percentage of failure (D \& F grades) in the class with and without MRS intervention. The figure reveals 
significant decline in course failing when using the MRS software along with interactive exercises. The data also reveals that, on average the number of A's in the class increased by $10 \%$ with intervention. Hence, in the case of students who had difficulty understanding concepts such as Truth Table and K-Map, the use of MRS software significantly enhances student learning.

Figure 5 and 6 shows the student performance data for K-Map and truth table exercises with and without using MRS. The figures represent the performance data averaged over the number of exercises and clearly represents the improvement when MRS is deployed in classroom. The data verifies that the use of the MRS software is effective in increasing student understanding of concepts that are typically difficult for students to comprehend.

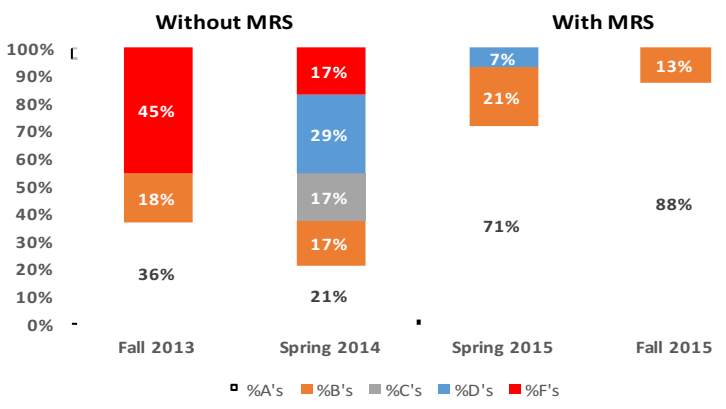

Figure 6. Grade distribution of truth table exercises.

In order to assess student perception of the MRS environment and its overall effectiveness, an experience survey was offered to the students during both intervened semesters. The survey results are shown in Table 3. This survey also reveals the impact of continuous improvement and fine-tuning of the proposed effort which is evident from notable increase in learner's positive impression from Spring 2015 to Fall 2015 in most cases.

A focus group was formed including a small number of students to further assess the effectiveness of the proposed approach. The participating students were asked specific questions about their experiences with the software and the improvements could be made to the system. All students positively attested that hands-on activities enhanced learning and MRS software was very effective in this regard. Anonymous question and immediate grading were appreciated most during the conversation. One focus group member mentioned that the anonymous question/vote feature should be utilized in each and every day of class and in other courses throughout the university. By using this feature of the MRS software, students feel they can let the instructor know whether he/she is going too fast or whether some concepts need more explanation without revealing their identity. Some students feel that making comments to the instructor about their teaching could potentially have an adverse impact on their grades. However, with the software, this fear can be alleviated. When asked what improvements could be made to the system, students

Table 3. Data on student perception about MRS.

\begin{tabular}{|c|r|c|c|c|c|}
\hline Question & Semester & $\begin{array}{c}\text { Strongly } \\
\text { agree }\end{array}$ & Agree & Disagree & $\begin{array}{c}\text { Strongly } \\
\text { disagree }\end{array}$ \\
\hline \multirow{2}{*}{$Q 1$} & Spring 2015 & $55 \%$ & $45 \%$ & & \\
\hline & Fall 2015 & $100 \%$ & & & \\
\hline \hline \multirow{2}{*}{$Q 2$} & Spring 2015 & $55 \%$ & $45 \%$ & & \\
\hline \hline \multirow{2}{*}{$Q 3$} & Fall 2015 & $100 \%$ & & & \\
\hline \hline \multirow{2}{*}{$Q 4$} & Spring 2015 & $73 \%$ & $27 \%$ & & \\
\cline { 2 - 6 } & Fall 2015 & $86 \%$ & $14 \%$ & & \\
\hline \hline \multirow{2}{*}{$Q 5$} & Spring 2015 & $55 \%$ & $45 \%$ & & \\
\hline \hline \multirow{2}{*}{$Q 6$} & Fall 2015 & $100 \%$ & & & \\
\cline { 2 - 6 } & Spring 2015 & $45 \%$ & $55 \%$ & & \\
\hline \hline \multirow{2}{*}{$Q 7$} & Fall 2015 & $86 \%$ & $14 \%$ & & \\
\hline & Fall 2015 & $100 \%$ & & & \\
\hline \hline \multirow{2}{*}{$Q 8$} & Spring 2015 & & $9 \%$ & $73 \%$ & $18 \%$ \\
\hline & Fall 2015 & $14 \%$ & & $43 \%$ & $43 \%$ \\
\hline & Fall 2015 & $14 \%$ & & $14 \%$ & $71 \%$ \\
\hline
\end{tabular}

Q1. MRS is helpful in understanding k-map and truth table.

Q2. MRS helps in visualizing the steps in k-map and truth table.

Q3. Seeing my grades immediately after taking the exercises is really helpful.

Q4. The k-map and truth table Apps are intuitive and easy to use.

Q5. I feel competent and confident to solve problems in MRS than in a pen-and paper-based setting.

Q6. I am enjoying this experience.

Q7. Using MRS did not improve my understanding of k-map and truth table problems.

Q8. Learning to use MRS software and related Apps is additional work beyond the normal course work.

requested that a) the software be used more often; b) it be made available for download onto their smartphones including iPhones; and c) more interactive exercises be developed to cover other concepts.

\section{RELATED WORK}

To facilitate interactive teaching and learning, 'clickers' were utilized in various ways [15]-[17], however, they are not suitable for assessing multi-step exercises which limits its applicability to only certain assessments. There is a plethora of research [3]-[7] which shows that mobile learning engages students more and improve student learning. For instance, Researchers at Rochester Institute of Technology reported that their use of a technologyrich learning environment in several undergraduate engineeringtechnology courses has improved learning and decreased withdrawals from, or failing grades in, the courses [3]. Boston University adopted tablet-based problem solving exercises in their freshman mathematics class and reported noticeable increase in student attendance and course completion [4]. Many other approaches [5]-[7] also reported enhanced educational experiences when technology such as mobile and tablet devices has been adopted in the classroom. However, the abovementioned efforts neither provide support for interactive exercises nor its assessment. Few researches support integrated assessment of interactive exercises. TRAKLA2 [18] is the pioneering learning environment that supports algorithm simulation exercises, 
automatic assessment and visual feedback. JHAVÉ [19] is a java application that renders algorithm visualizations and allows student explorations by pop-up questions. JSAV [20] is a JavaScript based algorithm visualization library that supports proficiency exercises. However, all of them support content delivery via web browser and therefore utilized technologies such as HTML, Java, JavaSript etc. but none to our knowledge do so via mobile App like MRS. The novelty of MRS is represented by its support for interactive problem solving, evidence-based teaching, anonymous classroom interaction and easier extendibility to other areas.

\section{CONCLUSION}

This paper presents details of MRS software, associated pedagogy, and lessons learned from using it in class. MRS provides instructors with the opportunity of evidence-based teaching by offering students with exercises having different learning outcomes and by receiving an immediate feedback on their performance. MRS is easy-to-use, extensible and can render interactive exercise developed by third party developer. The MRS software, associated interactive exercise Apps along with documentation and installation information are available for download from [21]. The assessment data verified that the MRS software is a really helpful intervention. Data collected on the distribution of the grades on specific assignments indicated that the use of the MRS software was very effective in increasing student understanding of concepts that are typically difficult for students to comprehend. Moreover, the students really enjoyed using the anonymous question feature as it allowed them to ask question without raising their hands. Additionally, these students were pleased that the software was able to provide immediate grading and feedback. Overall, student experiences with the system was positive and students enjoyed using it. Starting from Spring 2016, MRS will be deployed in two of the CS courses, where all of the seven developed interactive exercise Apps will be administered and in Fall 2016, MRS will also be used in 3 other disciplines alongside Computer Science. It would be interesting to see results obtained when using the software for additional courses, particularly those outside of the Department of Computer Science. With the advent of the MRS software, there is a tremendous opportunity to enhance the learning of hundreds, even thousands of students.

\section{ACKNOWLEDGMENTS}

This research was supported by National Science Foundation Award \# 1332531.

\section{REFERENCES}

1. D. Perry, How The Brain Learns Best, Instructor Magazine, 11: 34-37, 2000.

2. T. L. Naps, G. Ro Bling, V. Almstrum, W. Dann, R. Fleischer, C. Hundhausen, A. Korhonen, L. Malmi, M. McNally, S. Rodger, and J. A. Vela' zquez-Iturbide. Exploring the role of visualization and engagement in computer science education. In Working group reports from ITiCSE'02 on Innovation and technology in computer science education, 131-152, 2002.

3. M. Cometa, Use of Technology-Rich Learning Environment Reveals Improved Retention Rates, Rochester University of Technology, Nov 16, 2011 http://www.rit.edu/news/ story.php?id $=48699$.

4. C. A. Romney, Tablet PC use in freshman mathematics classes promotes STEM retention, Frontiers in Education Conference (FIE), F1J-1 - F1J-7, 2011.
5. Z. Avery, M. Castillo, H. Guo, J. Guo, N. Warter-Perez, D. S. Won, J. Dong, Implementing Collaborative Project-Based Learning using the Tablet PC to enhance student learning in engineering and computer science courses, Frontiers in Education Conference, F1E-1-F1E-7, 2010.

6. R. J. Young, Mobile College App: Turning iPhones Into 'Super-Clickers' for Classroom Feedback, Chronicle of higher education, 2008.

7. D. Berque, An evaluation of a broad deployment of DyKnow software to support note taking and interaction using penbased computers. Journal of Computing Sciences in Colleges, 21(6): 204-216, 2006.

8. J. Hattie, Visible learning: A synthesis of over 800 metaanalyses relating to achievement. Routledge, 2000.

9. G. Wiggins, Seven keys to effective feedback. Educational Leadership, 70: 10-16, 2012.

10. M. M. Fuad, D. Deb, J. Etim, and C. Gloster, Mobile Response System: A Novel Approach to Deliver Interactive and Hands-on Activity in the Classroom, Journal of Educational Technology Research and Development, Springer, Under revision, 2016.

11. D. Deb, M. M. Fuad and W. Farag, Developing Interactive Classroom Exercises for use with Mobile Devices to Enhance Class Engagement and Problem-solving Skills, IEEE Frontier's of Education Conference, IEEE Press, 343346, Madrid, Spain, October 22-25, 2014.

12. M. M. Fuad, D. Deb. and J. Etim, An Evidence Based Learning and Teaching Strategy for Computer Science Classrooms and its Extension into a Mobile Classroom Response System, Proceedings of the 14th IEEE International Conference on Advanced Learning Technologies (ICALT), IEEE Press, 149-153, Athens, Greece, July 7-9, 2014.

13. T. Postmes, R. Spears, K. Sakhel, and D.De Groot, Social influence in computer-mediated communication: The effects of anonymity on group behavior, Personality and Social Psychology Bulletin, 27: 1243-1254, 2001.

14. M.-T. Félix, C.-O. Jesús, G.-J. Luis, Anonymity effects in computer-mediated communication in the case of minority influence, Computers in Human Behavior, 23:1660-1674, 2007.

15. J. E. Caldwell, Clickers in the Large Classroom: Current Research and Best-Practice Tips, $C B E$ - Life Sciences Education, 6(1): 9-20, 2007.

16. J. K. Knight and W. B. Wood, Teaching more by lecturing less, Cell biology education, 4(4): 298-310, 2005.

17. E. E. Fredericksen and M. Ames, Can a $\$ 30$ Piece of Plastic Improve Learning? An Evaluation of Personal Responses Systems in Large Classroom Settings, EDUCAUSE, 2009.

18. L. Malmi, V. Karavirta, A. Korhonen, J. Nikander, O. Seppa la", and P. Silvasti. Visual algorithm simulation exercise system with automatic assessment: TRAKLA2. Informatics in Education, 3(2):267-288, 2004.

19. T. L. Naps, Jhavé: Supporting algorithm visualization, Computer Graphics and Applications, IEEE, 25(5):49-55, 2005.

20. V. Karavirta and C. A. Shaffer, Creating Engaging Online Learning Material with the JSAV JavaScript Algorithm Visualization Library, in Learning Technologies, IEEE Transactions on, $\mathrm{PP}(99)$ : 1-1, 2015.

21. Mobile Response System, http://compsci.wssu.edu/MRS, 2016. 